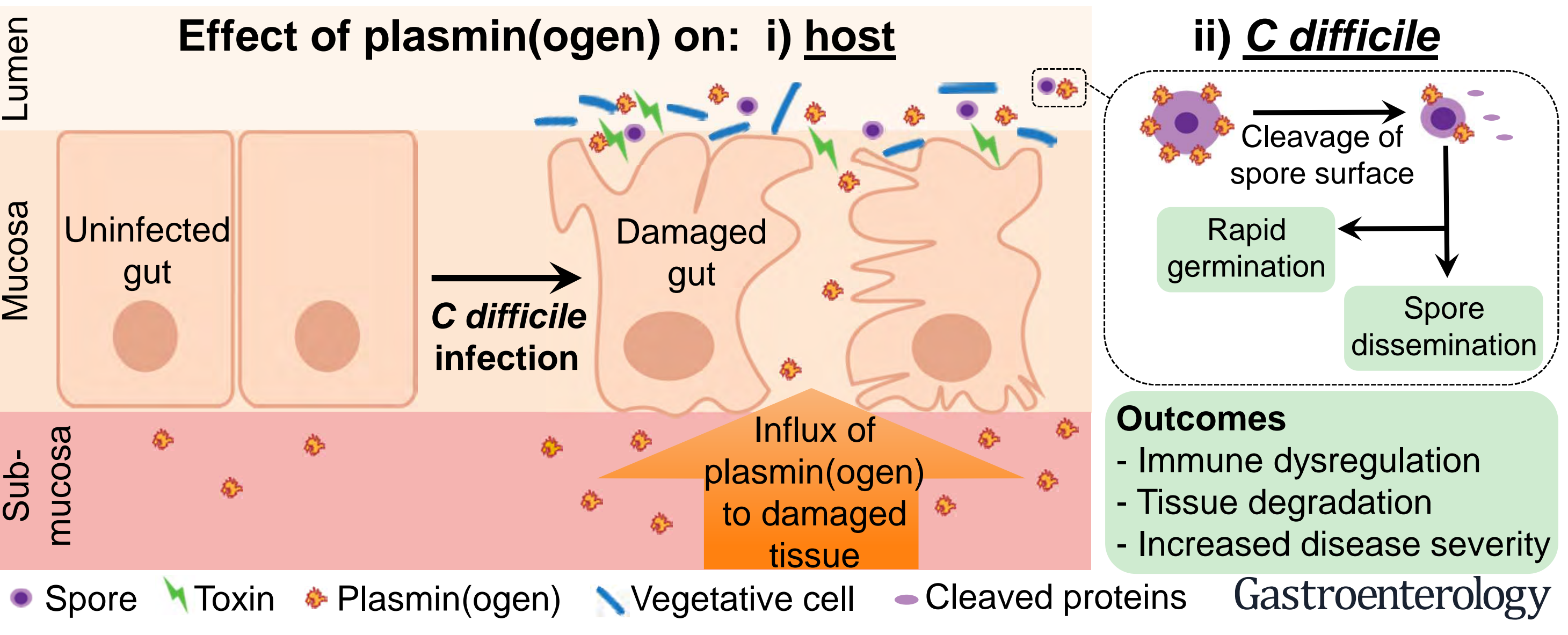




\section{Human Plasminogen Exacerbates Clostridioides difficile Enteric Disease and Alters the Spore Surface}

Short title: Human plasminogen and $C$ difficile infection

Authors: M. M. Awad ${ }^{1} \dagger$, M. L. Hutton ${ }^{1} \dagger$, A. J. Quek ${ }^{4} \dagger$, W. P. Klare ${ }^{2}$, S. J. Mileto ${ }^{1}$, K. Mackin $^{1}$, D. Ly ${ }^{3}$, V. Oorschot ${ }^{4,6}$, M. Bosnjak ${ }^{1}$, G. Jenkin ${ }^{11}$, P. J. Conroy ${ }^{4}$, N. West ${ }^{5}$, A. Fulcher $^{6}$, A. Costin ${ }^{4}$, C. J. Day ${ }^{7}$, M. P. Jennings ${ }^{7}$, R. L. Medcalf ${ }^{8}$, M. Sanderson-Smith ${ }^{3}$, S. J.

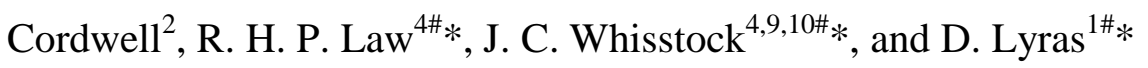

Affiliations: ${ }^{1}$ Infection and Immunity Program, Monash Biomedicine Discovery Institute and Department of Microbiology, Monash University, Clayton VIC 3800, Australia. ${ }^{2}$ School of Life and Environmental Sciences and Charles Perkins Centre, The University of Sydney, Sydney NSW 2006, Australia. ${ }^{3}$ School of Biological Sciences, University of Wollongong and Illawarra Health and Medical Research Institute, Wollongong NSW 2500, Australia. ${ }^{4}$ ARC Centre of Excellence in Advanced Molecular Imaging and Biomedicine Discovery Institute, Department of Biochemistry, Monash University, Clayton VIC 3800, Australia. ${ }^{5}$ School of Chemistry and Molecular Biosciences and Australian Infectious Diseases Research Centre, University of Queensland, St. Lucia QLD 4072, Australia. ${ }^{6}$ Monash Micro Imaging (MMI), Monash University, Clayton, VIC 3800, Australia. ${ }^{7}$ Institute for Glycomics, Griffith University, Gold Coast, QLD 4222, Australia. ${ }^{8}$ Molecular Neurotrauma and Haemostasis, Australian Centre for Blood Diseases, Monash University, Clayton VIC 3800, Australia. ${ }^{9}$ EMBL Australia, Monash University, Clayton VIC 3800, Australia. ${ }^{10}$ South East UniversityMonash Joint Institute, Institute of Life Sciences, Southeast University, Nanjing 210096, 
China. ${ }^{11}$ Monash Infectious Diseases, Monash Health, Clayton, VIC, 3168, Australia.

$\dagger$ These authors contributed equally to the work and should be regarded as joint first authors

\# These authors contributed equally to the work and should be regarded as joint senior authors

Grant support: RLM is supported by a National Health and Medical Research Council of Australia (NHMRC) Principal research Fellowship. MPJ is supported by NHMRC Program Grant 1071659 and Principal Research Fellowship 1138466, and is also supported by NHMRC project grant 1045755 awarded to RLM and NHMRC project grant awarded to MLS. JCW is supported by an NHMRC Senior Principal Research Fellowship, APP1127593. He acknowledges the previous support of an Australian Research Council (ARC) Federation Fellowship. This project was supported by NHMRC grant 1107969 and an ARC Future Fellowship FT120100779 awarded to DL.

Abbreviations: CDI, $C$ difficile infection; CPE, cytopathic effect; EACA, aminocaproic acid; FCS, fetal calf serum; hPLG, human plasminogen; MES, (2-(N-morpholino)ethanesulfonic acid); mPLG, mouse plasminogen; PEG, polyethylene glycol; PBS; phosphate buffered saline; STED, stimulated emission depletion; TA, tranexamic acid TFA, trifluoroacetic acid; tPA, tissue plasminogen activator; uPA, urokinase plasminogen activator.

*Correspondence to: ruby.law@monash.edu, james.whisstock@monash.edu, dena.1yras@monash.edu

Declaration of interests: The authors declare no competing interests.

\section{Writing Assistance: None}


Author Contributions: Conceptualization, D.L., M.M.A., M.L.H.; Methodology, M.M.A., M.L.H., A.J.Q., W.P.K., P.J.C., A.F., A.C., C.J.D., D.L., J.C.W., S.J.C., M.P.J; Formal Analysis, M.M.A., M.L.H., A.J.Q., W.P.K., S.J.C.; Investigation, M.M.A., M.L.H., A.J.Q., W.P.K., S.J.M., K.M., A.F., V.O., C.J.D. P.J.C.; Resources, D.L., D.Ly, M.B., G.J., R.H.P.L., N.W., M.P.J., R.L.M., M.S-S, S.J.C., J.C.W.; Writing, Original Draft, M.M.A., M.L.H., D.L.; Writing, Review and Editing, D.L., J.C.W., M.M.A., M.L.H., R.L.M., M.S-S., R.H.P.L; Visualization, M.M.A., M.L.H., A.J.Q., W.P.K.; Supervision, D.L. J.C.W., R.H.P.L., S.J.C; Funding Acquisition, D.L., R.L.M., M.P.J., J.C.W.

\begin{abstract}
Background \& Aims: The protease plasmin is an important wound healing factor, but it is not clear how it affects gastrointestinal infection-mediated damage, such as that resulting from Clostridioides difficile. We investigated the role of plasmin in $C$ difficile-associated disease. This bacterium produces a spore form that is required for infection, so we also investigated the effects of plasmin on spores.
\end{abstract}

Methods: C57BL/6J mice expressing the precursor to plasmin, the zymogen human plasminogen (hPLG), or infused with hPLG, were infected with $C$ difficile and disease progression was monitored. Gut tissues were collected and cytokine production and tissue damage were analyzed using proteomic and cytokine arrays. Antibodies that inhibit either hPLG activation or plasmin activity were developed and structurally characterized and their effects were tested in mice. Spores were isolated from infected patients or mice and visualized using super-resolution microscopy; the functional consequences of hPLG binding to spores were determined. 
Results: hPLG localized to the toxin-damaged gut, resulting in immune dysregulation with an increased abundance of cytokines (such as IL1A, IL1B, IL3, IL10, IL12B, MCP1, MP1A, MP1B, GCSF, GMCSF, KC, TIMP-1), tissue degradation, and reduced survival. Administration of antibodies that inhibit plasminogen activation reduced disease severity in mice. $C$ difficile spores bound specifically to hPLG and active plasmin and degraded their surface, facilitating rapid germination.

Conclusions: We found that hPLG is recruited to the damaged gut, exacerbating $C$ difficile disease in mice. hPLG binds to $C$ difficile spores, and, upon activation to plasmin, remodels the spore surface, facilitating rapid spore germination. Inhibitors of plasminogen activation might be developed for treatment of $C$ difficile or other infection-mediated gastrointestinal diseases.

Key Words: pathogenic bacteria, microbe, fibrinolytic system, liver enzyme

\section{Introduction}

Human proteases are one of the largest known enzyme families and are involved in many functions including the regulation of multiple signaling pathways essential for maintaining homeostasis ${ }^{1}$. Plasmin, the active form of the liver-produced plasminogen, is one such mammalian serine protease, with roles in fibrinolysis, cell migration and tissue remodeling, including the degradation of basement membranes and the extracellular matrix (ECM) ${ }^{2,3}$. Many pathogens, including Group A Streptococcus and Bacillus anthracis, exploit the fibrinolytic system by recruiting and activating plasminogen to the microbe surface ${ }^{4,5}$. Although it is the vegetative form of pathogenic bacteria that usually bind to plasminogen, in B. anthracis the spore form was found to bind plasminogen, albeit without consequence for disease severity or virulence ${ }^{5}$. As a consequence of its ability to degrade host tissues, 
microbe-associated plasmin greatly enhances bacterial invasion of the host, thereby increasing disease severity during infection ${ }^{2,6}$.

The role that plasminogen may play during infection of the gastrointestinal tract is not known. Gastrointestinal damage resulting from infections, autoimmune disorders, alcohol abuse, and burns often causes enteric tissue permeability which can result in the escape of resident bacteria, pathogens and other microbial components from the gut lumen into other tissues that are not normally exposed to these products ${ }^{7}$. The localization of these factors beyond the gastrointestinal epithelium induces an inflammatory host response which results in the infiltration of immune cells and other host factors such as cytokines and proteases into the affected underlying tissue ${ }^{7,8}$. Under normal conditions plasminogen, which is most abundant in the circulatory system, is in low abundance in the gastrointestinal tract ${ }^{9}$ but the effect of tissue damage on enteric plasminogen levels and function is unknown.

Clostridioides difficile infects the gut and causes severe tissue damage through the production of potent exotoxins ${ }^{10}$. A key driver of $C$ difficile infection (CDI) relates to the ability of this bacterium to form an inert, and highly robust, spore form which allows survival of the bacterium in hostile environments ${ }^{10}$. Spores initiate and transmit disease, and contribute to disease relapse - an event that occurs in up to $30 \%$ of patients ${ }^{11}$. In the colon, spores germinate into vegetative cells that colonize the gut and produce up to three toxins, the large clostridial toxins TcdA and TcdB and the $C$ difficile transferase (CDT) toxin, which act directly to damage and permeabilize colonic tissue through their effects on the actin cytoskeleton of host cells ${ }^{12}$. C difficile vegetative cells also produce spores, which are responsible for transmitting disease and perpetuating the infection cycle ${ }^{10}$.

We hypothesized that as a consequence of its role in wound healing, plasminogen may be recruited to the site of infection where it may exacerbate or inhibit disease progression. Here, 
we show that human plasminogen (hPLG) exacerbates $C$ difficile disease in a mouse infection model, in synergy with toxin-mediated enteric damage. The gastrointestinal damage that occurs during infection leads to an increased abundance of hPLG in gastrointestinal tissues, which has several downstream consequences, including augmented inflammation, tissue degradation and dissemination of spores beyond the infection site, and coincides with poorer disease outcomes in infected mice. We also show that $C$ difficile spores bind specifically to hPLG which changes the phenotypic properties of these otherwise largely dormant cells. Binding and activation of plasminogen changes the spore surface, resulting in a thinner outer spore layer and an increase in spore germination capacity. This study therefore provides evidence of a mechanism by which an enteric pathogen can dysregulate the host plasminogen system to intensify gastrointestinal disease.

\section{Materials and Methods}

\section{C difficile mouse models}

Animal handling and experimentation were performed in accordance with Victorian State Government regulations and approved by the Monash University Animal Ethics Committee (Monash University AEC no. SOBSB/M/2010/25 or MARP/2014/136). For mice and experimental design, tissue processing and scoring, see Supplementary Material.

\section{Cytokine arrays on mouse gut tissue}

Cecal tissue was homogenized through $100 \mu \mathrm{m}$ nylon cell strainers in lysis buffer $[0.1 \%$ Triton-X 100 in PBS, with one protease inhibitor cocktail tablet/20ml (cOmplete ${ }^{\mathrm{TM}}$, EDTAfree Roche)], centrifuged at $13,000 \times \mathrm{g}$ for $5 \mathrm{mins}$ at $4^{\circ} \mathrm{C}$ and supernatants adjusted to 200 $\mu \mathrm{g} / \mathrm{ml}$ prior to analysis via a modified 22-plex mouse ProcartaPlex custom kit (eBioscience) and a mouse TIMP-1 ELISA (eBioscience). Assays were conducted according to 
manufacturer's instructions; Cytokine levels measured using the Bio-Plex MAGPIX multiplex reader (BIO-RAD); Results tabled using the ProcartaPlex Analyst 1.0 software program (eBioscience). Statistical analysis: Mann-Whitney U test (Two-tailed).

\section{Proteomics analysis using LC-MS/MS}

Equal dry weight of cecum was pooled per experimental condition ( $n=3-5$ replicates). Tissue lysis and processing for LC-MS/MS is described in Supplementary Material. Raw data analysed with MaxQuant (v 1.6.0.16) against the UniProt mouse proteome (release 12/6/17, 52015 proteins) and post-processing of significantly changing proteins were performed as described in Supplementary Material. A minimum of three independent biological and technical mass spectrometric replicates were performed.

PLG extraction from mouse gut tissue

PLG was extracted from mouse gut tissues as before ${ }^{16}$, with some modifications (See Supplementary Material).

\section{Spore preparation}

C difficile spores were prepared using method described previously ${ }^{17}$ except that spores were harvested from TY plates ${ }^{18}$ and washed in ice-cold sterile $\mathrm{dH}_{2} \mathrm{O}$ until supernatants became clear (8-10 times).

\section{Isolation of fecal spores}

Spores were isolated from fecal samples collected from two $C$ difficile-infected human subjects or from $C$ difficile-infected mice. Samples were resuspended in PBS, filtered through a $100 \mu \mathrm{m}$, then $70 \mu \mathrm{m}$ nylon cell strainer (Falcon) and centrifuged ( $1 \mathrm{~min}$ at $14,000 \times \mathrm{g}$ at 
RT). Pellets were washed 5 times in ice-cold sterile $\mathrm{dH}_{2} \mathrm{O}$ then further purified using Histodenz ${ }^{17}$.

\section{Detecting hPLG binding to $C$ difficile spores via surface plasmon resonance}

Spores were immobilized to the surface of $\mathrm{C} 1$ series sensor chips (GE Health Care) following the C1 NHS/EDC method template with a contact time of 900 seconds at a flow rate of 5 $\mu 1 / \mathrm{min}$ in $10 \mathrm{mM}$ sodium acetate, $\mathrm{pH} 5.5$. Interaction of human, equine, porcine or mouse plasminogen (A95350M; Life Science, Inc.) with spores was determined using concentrations of $0.16-100 \mu \mathrm{g} / \mathrm{ml}$ and using single cycle kinetics in $1 \mathrm{x}$ PBS at $20 \mu \mathrm{l} / \mathrm{min}$ with a 60 second contact time and a final dissociation time of 10 mins.

Detection of PLG binding via Western blot analysis

Detection of hPLG bound to $C$ difficile spores from strains M7404 ${ }^{14}$, R20291 ${ }^{19}$, JGS6133 ${ }^{20}$, AI35 ${ }^{21}$, DLL3109 ${ }^{22}$, VPI10463 ${ }^{23}$ and CD37 ${ }^{24}$ was performed as before ${ }^{5}$ except that $10 \mu \mathrm{g}$ hPLG (Banksia Scientific) was bound to $1 \times 10^{6}$ spores. Binding was analyzed via Western blot using 1-2 $\mu \mathrm{g} / \mathrm{ml} \alpha$-human Plasmin(ogen) antibody (MAB2596; R\&D Systems) and detected using a goat $\alpha$-mouse IgG $(\mathrm{H}+\mathrm{L})$ HRP conjugated antibody (Millipore) with the ChemiDoc XRS+ system (Biorad).

\section{PLG binding detection via Immunofluorescence}

$1 \times 10^{9} \mathrm{C}$ difficile spores were incubated with $10 \mu \mathrm{g}$ hPLG as before ${ }^{5}$. hPLG bound spores were then incubated in 5\% skim milk $(1-2 \mathrm{~h} / \mathrm{RT})$, washed 3 times in TBS buffer $(5 \mathrm{mM}$ Tris-HCI, $15 \mathrm{mM} \mathrm{NaCI}, \mathrm{pH}$ 7.4), incubated with $2 \mu \mathrm{g} / \mathrm{ml} \alpha$-human Plasmin(ogen) antibody (overnight $/ 4^{\circ} \mathrm{C}$, washed again followed by incubation with an Alexa Fluor 488 goat $\alpha$-mouse $\operatorname{IgG}(\mathrm{H}+\mathrm{L})$ (Life Technologies) (45 mins/RT). After the final washes, spores were 
resuspended in TBS buffer, mounted onto poly-L-lysine slides (Thermo Scientific) and imaged on an Olympus BX-51 attached to an Olympus power unit U-RFL-T.

PLG binding detection via super resolution microscopy (STED)

hPLG was labeled with Alexa Fluor 647 NHS ester (Invitrogen, A20006) as per manufacturer's instructions (GE Healthcare, 17-0853-02). Spores were bound with $10 \mu \mathrm{g}$ of labeled hPLG, washed 3 times as before, and incubated (overnight/ $4^{\circ} \mathrm{C}$ ) with an anti- $C$ difficile whole spore antibody ${ }^{25}$ labeled with either Atto-488 NHS ester (Invitrogen, 41698) or Alexa Fluor 568 NHS ester (Invitrogen, A20003), as above. Following 3 washes, spores were mounted onto poly-L-lysine coated coverslips (No.1.5H, Zeiss) and imaged using a stimulated emission depletion (STED; Abberior Instruments GmbH, Göttingen, Germany) microscope equipped with an Olympus 100x oil objective (UPlanSApo NA=1.4) with a 1 watt $775 \mathrm{~nm}$ pulsed STED laser. Imagine acquisition was performed in RESCue (REduction of State transition Cycles) mode (775 STED laser power of 6\%/ confocal laser power for 640 $\mathrm{nm}$ of $60 \%$ ). For $488 \mathrm{~nm}$ only a confocal laser of $20 \%$ was used. Unlabeled spores and spores bound to unlabeled hPLG were used as negative controls.

Bone marrow derived macrophage (BMDM) isolation and infection

BMDM isolation was performed as before ${ }^{26}$. Cells were serum starved overnight prior to infection with M7404 spores that were either untreated or plasmin treated as before (MOI 10:1). TEM imaging and analysis was performed $(n=60 /$ group) as described below

TEM imaging of untreated versus plasmin-bound treated spores 
$1 \times 10^{9}$ M7404 spores were either untreated or plasmin treated as before. TEM preparation and imaging of spores were as before ${ }^{27}$. Sections were imaged on a JEM-1400Flash (Jeol). Statistical analysis: Mann-Whitney U test (Two-tailed).

Germination assay of untreated spores versus plasmin-bound spores

Germination assays were performed as before ${ }^{28}$ and are detailed in Supplementary Material. Data represent the mean of at least 3 biological replicates. Statistical analysis: Two-way ANOVA with Tukey's multiple comparisons test.

\section{PLG activation assay}

Using a modified protocol ${ }^{29}, 1 \times 10^{9}$ untreated spores or spores bound with $10 \mu \mathrm{g}$ hPLG (see Western blot protocol except washes were carried out in $0.01 \%$ TBS-Tween 80) were incubated with or without either $0.001 \mu \mathrm{M}$ huPA (R\&D Systems), human tissue plasminogen activator (htPA) (Millipore) or mouse uPA (muPA) (Molecular Innovations) in the presence of $100 \mu \mathrm{M}$ of S-2251 (Chromogenix). Experiments were conducted independently 3 times, with a representation of the results shown.

\section{PLG extraction from mouse gut tissue}

Cecum was collected from five hPLG or PBS infused mice, the tissue from each group pooled, weighed and passed through $100 \mu \mathrm{m}$ nylon cell strainers (Falcon), Suspensions were then centrifuged $\left(4^{\circ} \mathrm{C} / 5 \mathrm{mins} / 13,000 \mathrm{x} \mathrm{g}\right)$ and the supernatants collected. The sample amount loaded onto a $12 \%$ SDS PAGE gel was based on the equivalent mg of pooled cecal tissue, and the extracted PLG visualized via Western blot analysis.

Germination assay of untreated spores versus plasmin-bound spores 
$2 \times 10^{7}$ untreated M7404 spores or plasmin-bound spores (PLG binding as per Western blot protocol, except that $0.001 \mu \mathrm{M}$ human urokinase plasminogen activator (huPA) was added at $37^{\circ} \mathrm{C}$ for $2.5 \mathrm{~h}$ for PLG activation) were tested.

Crystallization of antibody and KR5-SP plasminogen fragment and micro plasmin (SP) binary complexes

Recombinant proteins were expressed and purified as previously described ${ }^{29}$. More details are provided in the Supplementary Material.

Impact of B10 and G05 on plasmin activity and hPLG activation

Plasmin activity and hPLG activation were carried out as before ${ }^{30}$

Binding of hPLG to antibody B10 and G05 measured by BiaCore T200

Binding experiments were performed as before ${ }^{30}$

\section{Results}

To test if plasminogen is recruited to the infection site, mice infused with, or transgenic mice expressing, hPLG were infected with a toxigenic wild-type (WT) strain of $C$ difficile (M7404) or a mutant strain (DLL3121) that no longer produces the major toxins, TcdA and TcdB ${ }^{12}$. WT-infected mice displayed epithelial damage, edema and inflammation in comparison to uninfected mice (Fig. 1A). We found that hPLG levels in the cecum of these WT-infected mice were significantly elevated in comparison to all controls (Fig. 1B). In addition, slightly elevated levels of hPLG were detected in the DLL3121 infected mice compared to uninfected animals (Fig. 1B). These results suggest that CDI and tissue damage leads to an influx of hPLG into the infected gastrointestinal tissue. The action of the major 
toxins TcdA and TcdB appears to be the main driver of this hPLG accumulation, although other bacterial factors, such as the $C$ difficile binary toxin (CDT) ${ }^{10}$, are sufficient to mediate partial hPLG influx. Collectively, these results suggest that CDI and tissue damage leads to an influx of hPLG into gastrointestinal tissue at the infection site.

To determine if hPLG tissue influx influences infection outcomes, we compared disease severity in WT C57BL/6J mice to that observed in transgenic mice that express hPLG, which were both infected with toxigenic $C$ difficile. Disease was greatly exacerbated when hPLG was present, with earlier disease onset seen in the hPLG-expressing mice as demonstrated by higher stool consistency scores (Fig. 1C), increased nest soiling (Fig. 1D), and poorer physiological conditions (Fig. 1E) (for scoring parameters see Supp. Tables $2-4$ ). The severity of the disease symptoms resulted in a significant reduction in survival time, with the hPLG transgenic mice requiring euthanasia 24 hours earlier than the WT mice (Fig. 1F). To ensure that disease exacerbation did not result from colonisation or toxin production differences between the groups of mice, feces were collected and analysed at 24 hours postinfection. TcdA and TcdB production was comparable across the two groups of mice (Fig. $1 \mathrm{G}, \mathrm{H})$, and no discernible difference in the numbers of spores shed from the mice was detected, with all mice shedding $5 \times 10^{6}-1 \times 10^{7}$ spores/g feces. Since TcdB is the major driver of tissue damage ${ }^{31}$, no difference in adherens junction integrity (Supp. Fig. 1G) or histopathological damage (Supp. Fig. 1H) was observed between cecal tissues from WT and hPLG transgenic infected mice.

We next investigated whether leakage and dissemination of gut luminal contents to distant organs had taken place in the infected animals. We reasoned that such an outcome may explain the observed disease exacerbation in hPLG mice. Accordingly, thymus, spleen and kidneys were harvested at 24 hours post-infection and examined for the presence of 
spores. Spores were detected in the kidney, spleen and thymus of the hPLG mice but not in C57BL/6J control mice, except for very low numbers in the thymus (Fig. 1I). Moreover, plasmin-bound spores exhibited an $\sim 3$-fold increase in association with bone marrow derived macrophages (BMDMs) ex vivo when compared to unbound spores (Supp. Fig. 2H), a mechanism that may facilitate extraintestinal spore dissemination.

An examination of cecal tissues harvested at 24 hours post-infection revealed significantly more inflammation (Fig. 1J) and immune cell influx (Supp. Fig1. H) in the hPLG mice compared to C57BL/6J mice. These findings were supported by experiments performed using mice infused with hPLG, which also exhibited accelerated disease symptoms (Supp. Fig. 1). The exacerbated disease seen in hPLG-expressing or infused mice was therefore reflected in the cecal pathology of the mice, suggesting that hPLG exacerbates gut inflammation during infection with toxigenic $C$ difficile which leads to more severe disease outcomes. The presence of hPLG in infected tissues also changed the host inflammatory profile, with significant increases in the production of cytokines detected in infected hPLG mice compared to infected PBS control mice (Fig. 2A). To confirm that altered cytokine expression did not result from the presence of hPLG alone, the cytokine levels in uninfected mice were examined. The levels in hPLG infused but uninfected mice were slightly, but not significantly, lower than those in the PBS infused uninfected mice (Fig. 2B), however, cytokine levels in both uninfected groups were low when compared to those in infected mice. A quantitative proteomic analysis of infected versus uninfected cecal tissues collected from PBS and hPLG infused mice supported these findings (Fig. 2C, D, E; Supp. Table 1). Taken together, these data suggest that $C$ difficile is able to recruit hPLG during the course of infection, and that the presence of this protease enhances inflammation and disease severity in the gut, in turn facilitating the translocation of $C$ difficile spores to extra-intestinal organs. 
Since the mice used in these studies all produce murine plasminogen (mPLG) in addition to $\mathrm{hPLG}^{4}$, we investigated the role of $\mathrm{mPLG}$ during infection with toxigenic $C$ difficile by comparing the disease outcomes of genetically modified mice that no longer express mPLG (mPLG KO) ${ }^{13}$ to unmodified C57BL/6J (WT) mice. No difference in disease progression, inflammation (Supp. Fig. 1F) or survival time (Fig. 1K) was observed between the two groups, suggesting that mPLG does not contribute to disease in this infection model.

As several diverse invasive pathogens recruit plasminogen onto their surface to enable host invasion and to subvert the host immune response ${ }^{5,6,32}$, we examined if $C$ difficile has the same capability. Unexpectedly, unlike most pathogens we discovered that hPLG did not bind to $C$ difficile vegetative cells (Fig. 3A), but instead bound strongly to spores, regardless of the origin (human or animal), geographic location or toxigenic status of the strain (Fig. 3A, C). Moreover, immunofluorescence (IF) and STED super resolution microscopy confirmed that hPLG bound to spores produced under laboratory culture conditions (Supp. Fig. 2A-C; Fig. 3D-F). Importantly, spores derived from $C$ difficile-infected patients (Fig. 3G-I) or $C$ difficile-infected mice (Fig. 3J-L) were also found to have hPLG on their surface, with STED imaging showing that hPLG bound in clusters around the spore surface in all cases (Fig. 3E, $3 \mathrm{H}, 3 \mathrm{~K})$. This result suggests that spores are naturally coated with hPLG within the context of the infected host.

Surface plasmon resonance confirmed that hPLG bound with high affinity to $C$ difficile spores $\left(\mathrm{KD}\right.$ of $13.4 \mathrm{nM} \pm 3.2 \mathrm{nM}, \mathrm{K}_{\mathrm{on}}$ of $55,520( \pm 6953) \mathrm{M} / \mathrm{s}$ and $\mathrm{K}_{\text {off }}$ of $7.3 \times 10^{-4}$ $\left.\left( \pm 1.1 \times 10-{ }^{4}\right) 1 / s\right)$ (Fig. 3B). Crucially, no concentration dependent interaction was detected between spores and mPLG (Fig. 3B). These data support the finding that no difference in virulence is seen between WT versus mPLG-deficient mice (Fig. $1 \mathrm{~K}$ ). C difficile spores also 
bound to porcine and equine PLG (Fig. 3B) although with lesser affinity than to human PLG, which may suggest that these proteins also contribute to disease exacerbation in such hosts.

The conversion of PLG to its most active form, plasmin, is tightly regulated ${ }^{3}$. PLG is converted to plasmin by host factors such as urokinase plasminogen activator (uPA) and tissue plasminogen activator (tPA) ${ }^{3}$. Accordingly, we performed PLG activation assays in the context of the spore-bound form. Unbound or spore-bound hPLG was incubated with human or murine uPA (huPA and muPA) and human tPA (htPA). Both the mouse and human factors activated hPLG to plasmin in either the unbound (Supp. Fig. 2D) or spore-bound state (Supp. Fig. 2E), suggesting that the spores have plasmin on their surface and can transport this active protease to any site that they occupy, within or outside the gut. This idea was supported by the proteomic analysis, which showed an increase in the amount of the mouse variants of the plasminogen-activating cascade only in infected hPLG mice (Fig. 2E), indicating that hPLG is activated and functional in the gastrointestinal tract of these animals.

Since plasmin has broad substrate specificity ${ }^{34}$, and to determine the functional outcome of plasminogen activation in the context of $C$ difficile spores, we investigated the spore structure to determine if it was altered, particularly on the surface. Strikingly, transmission electron microscopy showed that hPLG bound to spores and activated using huPA reduced the thickness of the exosporium, or the outermost spore layer, by half, from an average of $73.9 \mathrm{~nm}$ in untreated spores to $34.6 \mathrm{~nm}$ in hPLG-bound spores (Fig. 4A-E). As germinants must traverse the exosporial layer before they can activate spore germination, the thickness and density of this layer may influence the efficiency of the germination process in the host. Germination assays were therefore performed, in the presence of the host-derived bile acid germinant, taurocholate, showing that the plasmin-bound spores germinated more rapidly than untreated spores (Fig. 4F). This result shows that binding and activation of hPLG 
directly changes the structural and functional properties of $C$ difficile spores, leading to faster germination, which may contribute to the earlier disease onset and severe disease outcomes observed in the hPLG-mouse models described above.

To further investigate the functional consequence of plasmin accumulation on the spore surface, we compared the ability of plasmin-bound and unbound spores to modify host proteins. Plasmin-bound spores specifically cleaved the complement protein $\mathrm{C} 3 \mathrm{~b}$; a phenotype that was reversed in the presence of the plasminogen activation inhibitor, tranexamic acid (Supp. Fig. 2F) and also degraded the ECM protein, fibronectin (Supp. Fig. 2G). Plasmin-mediated modification of the surface of a bacterial cell, in this case a spore, has not been demonstrated before and brings a new understanding to how a pathogen has adapted to take advantage of the proteolytic activity of a host protein.

We next investigated whether targeting hPLG therapeutically may present an effective treatment for CDI. Previous studies have shown that the plasmin system is challenging to specifically inhibit, with small molecule plasmin inhibitors cross-reacting with other key proteases of the coagulation system ${ }^{35}$. To overcome these problems, we developed two monoclonal antibodies (G05 and B10) that specifically target the plasmin(ogen) system. Both antibodies bind to plasminogen (Supp. Fig. 3C-E). However, molecule G05 was found to effectively inhibit the generation of active plasmin (Supp. Fig. 3A) whilst molecule B10 was found to effectively inhibit the activity of plasmin (Supp. Fig. 3B). To understand the structural basis for the function of these molecules we determined the X-ray crystal structure of a scFv fragment of G05 in complex with a fragment (KR5-SP) of plasminogen (Fig. 4G; Supp. Table 5), and B10 in complex with micro-plasmin (Fig. 4H; Supp. Table 5). The G05 complex revealed that the antibody specifically interacts with the activation loop of hPLG, thus preventing activators such as tPA and uPA from cleaving and activating the zymogen. In 
contrast, the catalytic domain of the plasmin (SP) structure reveals that B10 directly binds to the plasmin active site, thus preventing substrate binding.

To test the efficacy of the antibodies, hPLG-infused C57BL/6J mice were infected with $C$ difficile and administered hepes buffer alone or a non-specific isotype-control (naïve) antibody as control groups, or antibody G05 or B10. Disease onset was not as rapid in the G05-treated group, with low scores detected at $24 \mathrm{~h}$ post-infection in both fecal consistency (Fig. 4I) and nest soiling (Fig. 4J) when compared to the control groups. The physiological appearance of G05-treated mice indicated a reduction in disease severity $24 \mathrm{~h}$ post-infection when compared to control groups (Fig. 4K). Strikingly, G05-treated mice also survived for 24 h longer than control mice (Fig. 4L). By contrast, the B10-treated group appeared similar to the infected control groups, with very little protection observed (Fig. 4I-L). These results suggest that administration of the plasmin inhibitor antibody G05 protects against severe disease, and prolongs the survival of infected mice.

\section{Discussion:}

This study demonstrates three key findings that may have broader implications for gut disease. Firstly, infection-mediated damage recruits plasminogen, a potent protease when activated to plasmin, to the gastrointestinal infection site, which exacerbates disease. Secondly, cleavage of spore surface proteins by plasmin accelerates germination, which is likely to exacerbate the infection cycle and contribute to higher disease severity. Thirdly, our work reveals a new disease mechanism in which an enteric pathogen exploits the host fibrinolytic system to exacerbate gastrointestinal disease.

The dysregulation or mislocalization of plasminogen contributes to the pathophysiology of inflammatory processes that can be detrimental to the host ${ }^{36}$. Here we 
have shown that hPLG exacerbates $C$ difficile-mediated disease, with disease progression occurring more rapidly in mice expressing hPLG than in mice that only express mouse PLG (C57BL/6J mice). The enteric damage that results from toxin-mediated CDI leads to approximately 3.25 times more hPLG in the cecum of hPLG infused and infected mice compared to hPLG infused but uninfected mice. Note that despite the finding that mPLG does not appear to contribute to disease in the infection model used in this study, additional work is needed to confirm that the function of hPLG is not influenced by the presence of mPLG.

Although the influx of immune-related cells and other factors into infection-damaged gastrointestinal tissue is well $\mathrm{known}^{8}$, the work described here has shown for the first time that a specific protease, hPLG, migrates into damaged gut tissue and exacerbates this infection-mediated damage, altering the immune response. Collectively, the results obtained from the gut histology, cytokine arrays and proteomics analysis demonstrate that a more pronounced neutrophil-driven response toward CDI occurs in the presence of hPLG, and correlates with the indirect recruitment of neutrophils that is stimulated by plasmin seen in non-infection studies ${ }^{36}$; this may serve to recruit additional hPLG to infected tissues since neutrophils bind to this host $\operatorname{protein}^{37}$.

As well as an increase in the numbers of immune cells recruited to the infection site, an increase in the expression of numerous pro-inflammatory markers and proteins involved in the recruitment and activation of neutrophils and macrophages, cell types which are hallmarks of CDI ${ }^{38}$, was detected in infected hPLG mice. Apart from worsening disease outcomes, this increase in immune cell influx might provide a mechanism for spore dissemination. Supporting this notion, B. anthracis spores can escape the lung through immune and epithelial cells to reach regional lymph nodes, from which they can further 
disseminate ${ }^{39}$. The increased association of plasmin-bound spores to BMDMs when compared to unbound spores in our study suggests a similar mechanism for spore dissemination may exist in CDI.

A reduction in the ECM component, fibronectin, was also detected in the infected cecum of hPLG mice. In other non-intestinal studies, PLG has been shown to proteolytically degrade ECM and basement membrane-related proteins, to assist with cell migration and to maintain host homeostasis ${ }^{3}$, and was also found to be an important initiator of systemic disease in group A streptococcal clone M1T1 ${ }^{6}$. In our study, an increase in the levels of complement related proteins in the cecum of infected hPLG mice was also detected, again suggesting that elevated levels of hPLG recruited to these tissues can alter the innate immune response. Other pathogens also degrade or reduce tissue integrity in the host through the recruitment of plasmin ${ }^{2}$. S. pneumoniae isolated from haemolytic uremic syndrome (HUS) patients, for example, has been shown to degrade fibronectin, cleave $\mathrm{C} 3 \mathrm{~b}$, and damage endothelial cells, which may serve to induce HUS-mediated pathology ${ }^{40}$. This phenotype has not, however, been previously demonstrated in the context of gastrointestinal pathogens. Here we show that $C$ difficile plasmin-bound spores are also able to degrade fibronectin and cleave $\mathrm{C} 3 \mathrm{~b}$, the latter of which may result in a reduced capacity for pathogen clearance by the host. The banding pattern is different to that observed when $\mathrm{C} 3 \mathrm{~b}$ is cleaved naturally in the host ${ }^{41}$, suggesting that hPLG cleavage either degrades this essential innate immune component or alters the usual pathway taken by $\mathrm{C} 3 \mathrm{~b}$ to initiate an innate immune response.

Our data show that the dysregulation and infiltration of hPLG in the gastrointestinal tract following toxin damage changes the dynamics of $C$ difficile infection and allow for a new model for CDI progression to be proposed. In this model, toxin-mediated gut damage recruits hPLG to the damaged region. The aberrant localization and activation of hPLG to 
plasmin in the wounded gut promotes further tissue damage through an inflammatory response that increases immune cell influx and degradation of host proteins. Once the infection is established, hPLG may be recruited to both ingested and newly produced spores, within the gut. Active plasmin remodels the spore surface, inducing rapid spore germination into toxin-producing vegetative cells and further enhancing disease severity, consistent with a role in accelerating disease. The plasmin-bound spores also alter the innate immune response, possess greater invasive potential and can be systemically spread throughout the host, which may increase the propensity for disease recurrence. Importantly, additional studies are required to examine how gut microbiota composition might be altered in the context of hPLG-infiltration since shifts in the post-antibiotic microbial balance may influence CDI disease or extraintestinal dissemination.

Excitingly, antibody G05, which inhibits plasminogen activation, successfully reduced disease severity, and is a promising adjunct therapy for current standard of care therapeutics in CDI. The G05 antibody has a short half-life (data not shown) and would need to be engineered for optimal luminal delivery and minimization of systemic effects; further optimization and rigorous clinical testing must therefore occur before this type of treatment can be used. Nevertheless, this approach of targeting the plasminogen / plasmin system is supported by the existing short-term clinical (extraintestinal) use of tranexamic acid, an FDA approved plasminogen activation inhibitor, which is currently used to reduce haemorrhaging in severe trauma and blood disorder patients ${ }^{42}$. Since several gastrointestinal pathogens mediate disease through toxigenic effectors which compromise tissue integrity, and many other gastrointestinal disorders result in similar leaky gut effects, our results have broader implications for our understanding of the mechanisms underlying enteric diseases and further raise the possibility that inhibition of human plasmin(ogen) may be of broad therapeutic utility. 


\section{Acknowledgements}

We thank Dr Tung-Liang (Tom) Chung from Crux Biolab for performing the tissue cytokine arrays, the Monash Histology Platform, Department of Anatomy and Developmental Biology, Clayton, Monash University and Monash Micro Imaging, Clayton, Monash University, for their technical assistance with pathology and confocal imaging, and Despina Kotsanas for collecting $C$ difficile human fecal specimens (Monash Infectious Diseases, Monash Health). WPK is supported by a Faculty of Science/University of Sydney Postgraduate Scholarship in Microbial Vaccinology. Proteomics analysis was supported by instrumentation accessed through Sydney Mass Spectrometry, a Core Facility of the University of Sydney.

\section{Legends}

Figure 1: hPLG is recruited to the CDI-damaged gut, exacerbates tissue damage and disease and aids in spore dissemination in the infected host. (A) Representative PAS/Alcian blue stained cecal sections from hPLG transgenic mice or hPLG infused WT mice that were either uninfected or infected with $C$ difficile M7404 WT or an M7404 toxin mutant (DLL3121; A-B-). Square brackets ([) indicate crypt hyperplasia, arrow heads ( $\mathbf{\Delta})$ represent epithelial damage, and asterisks $(*)$ represent edema and inflammation. Scale bar represents $200 \mu \mathrm{m}$. (B) Western blots showing the amount of hPLG extracted from the cecum of hPLG transgenic $C$ difficile uninfected versus infected mice. Purified hPLG is included as a positive control (lane 1). hPLG extracted from the cecum of uninfected hPLG (lane 2) and $C$ difficile infected hPLG mice (lane 3 ) and from the cecum of hPLG infused uninfected (lane 4), M7404 toxin mutant (A-B-) infected (lane 5) or M7404 infected C57BL/6J mice (lane 6) is shown. Units (100 and 75) refer to the molecular weight of protein standards, in $\mathrm{kDa}$. (C) Fecal consistency, (D) Cage appearance, (E) Physiological appearance and (F) Survival time for hPLG transgenic $(\mathrm{n}=20)$ and WT $(\mathrm{n}=13)$ C difficile M7404 infected mice, uninfected 
controls for hPLG $(\mathrm{n}=7)$ and WT $(\mathrm{n}=11)$ mice. $(\mathbf{G}, \mathbf{H})$ Fecal toxin levels from $C$ difficile infected hPLG transgenic mice or WT mice, assessed using HT29 cells (TcdA) (G) and Vero cells (TcdB). (H) No statistically significant differences in toxin levels were observed. (I) $C$ difficile spore numbers in the kidney, spleen and thymus of $C$ difficile infected hPLG transgenic mice or WT mice. (J) Cecal inflammation score of hPLG transgenic infected and uninfected mice. (K) Survival times for mPLG KO $(\mathrm{n}=18)$ and WT $(\mathrm{n}=18) C$ difficile M7404 infected mice, uninfected controls for mPLG KO $(n=5)$ and WT $(n=6)$ mice. Note that WT refers to C57BL/6J mice. Statistical analysis: One-way Anova with Tukey's multiple comparisons $(* * \mathrm{P}<0.005 ; * * * \mathrm{P}<0.0005 ; * * * * \mathrm{P}<0.0001)(\mathrm{C}, \mathrm{D}, \mathrm{E}, \mathrm{J})$ or a Mann Whitney test; $* \mathrm{P}<0.05 ; * * \mathrm{P}<0.001 ; * * * \mathrm{P}<0.0001)(\mathrm{F}, \mathrm{G}, \mathrm{H}, \mathrm{I}, \mathrm{K})$.

\section{Figure 2: hPLG alters the host inflammatory response and tissue integrity during CDI.}

(A) Cytokine levels using a 22-plex cytokine array and TIMP-1 ELISA on tissue lysates from the cecum of either PBS infused or hPLG infused $C$ difficile M7404 infected mice are shown. The fold ratio represents the cytokine levels present in the gut tissues of infected mice normalized to levels obtained from each respective uninfected mouse group. Only the cytokine levels that differed between the hPLG and PBS infused and infected mice are shown. (B) The abundance $\left(\log _{10}\right)$ of the same cytokines that differed between the uninfected hPLG infused and uninfected PBS infused mice are shown for the cecum of each uninfected mouse group. The cytokine levels in hPLG infused but uninfected mice were slightly, but not significantly, lower than those in the PBS infused uninfected mice, however, cytokine levels in both uninfected groups were low when compared to those in infected mice. For $(\mathbf{A}, \mathbf{B})$ statistical analysis: Mann Whitney test $(* \mathrm{P}<0.05 ; * * \mathrm{P}<0.001$; $* * * \mathrm{P}<0.0001)$. (C) Proteomics by label-based liquid chromatography coupled to tandem mass spectrometry (LC-MS/MS) 
identified an average of 36696 unique peptides that were quantified across all 4 treatment groups (PBS uninfected; hPLG uninfected; PBS infected; hPLG infected), and that represented 5041 protein groups. Of these, 4426 protein groups were identified in at least 2 of 3 replicates and were represented by $\geq 2$ peptides to allow relative quantification. Gene ontology (GO) biological process terms statistically over-represented (FDR $<0.05$, red line denotes significance cut-off) in hPLG infected mice, compared to PBS infected mice. A significant number of over-represented GO terms are associated with innate and adaptive immunological processes, and a cross-section were selected for further analysis. (D) Immunological and tissue processes of interest, including the plasminogen activating cascade (E), were selected from GO analyses. GO term-associated protein abundances across treatment groups were scaled using z-scores at the protein level. Lower z-score (dark blue) denotes lower abundance, whereas higher z-scores (green and yellow) denote higher abundance.

Figure 3: Human, equine and porcine but not murine PLG binds to $C$ difficile spores. (A) Western blot of $C$ difficile M7404 vegetative cells using an anti-human PLG Ab. Vegetative cells incubated in the absence (V) or presence of hPLG (VP) are indicated. (B) Representative Biacore sensor curves for the interaction between human, equine, and porcine PLG and M7404 spores and lack of interaction between mouse PLG and spores. Data were fit to a one site specific binding model. (C) Binding of hPLG to $C$ difficile spores from diverse locations and origins (M7404 Canadian human epidemic isolate (E1); R20291 UK human epidemic isolate (E2); JGS6133 US animal isolate (A-US); A135 Australian animal isolate (A-AU); DLL3109 Australian human epidemic isolate (E-AU); VPI10463 US human reference isolate (R) and CD37 US non-toxigenic isolate (NT)) and detected using an antihuman PLG Ab. For (A) and (C), Spores incubated in the absence (S) or presence of hPLG (SP) are indicated. Purified hPLG (positive control) protein (P) and supernatants from spores 
washed 5 times with binding buffer to remove any unbound PLG (W) are also included as controls. Units (100 and 75) refer to the molecular weight of protein standards, in kDa. (D-L) Stimulated emission depletion (STED) microscopy of in vitro derived (D-F), human derived fecal (G-I) and mouse derived fecal (J-L) $C$ difficile spores stained using an anti-spore antibody (green) bound to hPLG (red). Merged STED images are shown in F, I and L.

Figure 4: Plasmin binding to $C$ difficile M7404 spores remodels the spore surface and increases germination efficiency, and molecule G05, a plasminogen activation inhibitor, reduces disease severity. Transverse TEM sections of (A) untreated or (B) plasmin-bound spores. The boxed regions from (A) and (B) are shown enlarged in (C) and (D), respectively. (E) The average length $(\mathrm{nm})$ of the exosporium was measured from untreated and plasminbound spores ( $\mathrm{n}=40$ spores/group, with 5 measurements per spore). Statistical analysis: Mann-Whitney $\mathrm{U}$ test $(\mathrm{P}<0.0001)$. (F) Germination rates of untreated or plasmin-bound spores in the presence of $50 \mu \mathrm{M}$ sodium taurocholate. Untreated spores incubated in the absence of sodium taurocholate were included as a negative germination control. Data represents the ratio of the $\mathrm{OD}_{600}$ at each time point $\left(\mathrm{OD}_{600}(\mathrm{t})\right)$ over the $\mathrm{OD}_{600}$ at time 0 $\left(\mathrm{OD}_{600}\left(\mathrm{t}_{0}\right)\right.$ and is measured over time (mins). Statistical analysis: Two-way Anova with Tukey's multiple comparisons test $(\mathrm{P}<0.01$ for untreated spores versus plasmin-bound spores in the presence of germinant). (G) Cartoon representation of the crystal structure of G05 bound to a hPLG fragment (containing the kringle 5 and the serine protease domains, KR5SP) showing that G05 binds to the activation loop and KR5. KR5, activation loop, SP, G05 light chain (LC) and heavy chain (HC) are depicted as light blue, yellow, cyan, pink and magenta, respectively. The peptide bond (arrow) between residues R561 and V562 of hPLG must be cleaved during activation. Key intermolecular side-chain interactions observed between KR5-SP and G05 (dotted line square) are shown on the right panel. Hydrogen bonds and salt bridges are shown as dashed lines. $(\mathbf{H})$ Cartoon representation of the crystal structure 
of B10 bound to the SP domain showing that B10 binds to residue D646 of the catalytic triad (H603, D646 and A741; shown in yellow stick and labelled). SP, B10 light chain (LC) and heavy chain (HC) are depicted as cyan, pink and magenta, respectively. Key intermolecular side-chain interactions observed between SP and B10 (dotted line square) are shown on the right panel as before. (I) Fecal consistency, (J) Cage appearance (K) Physiological appearance and (L) Survival curve of hPLG infused and $C$ difficile M7404 infected mice either injected with hepes (blue circles), B10 antibody (orange squares), G05 antibody (green triangles) or a naïve (non-specific isotype-control) anti-chicken antibody (purple upsidedown triangles). Statistical analysis: Mann Whitney test; $* \mathrm{P}<0.05$.

\section{References}

1. Marshall NC, Finlay BB, Overall CM. Sharpening Host Defenses during Infection: Proteases Cut to the Chase. Mol Cell Proteomics 2017;16:S161-S171.

2. Lahteenmaki K, Edelman S, Korhonen TK. Bacterial metastasis: the host plasminogen system in bacterial invasion. Trends Microbiol 2005;13:79-85.

3. Law RH, Abu-Ssaydeh D, Whisstock JC. New insights into the structure and function of the plasminogen/plasmin system. Curr Opin Struct Biol 2013;23:836-41.

4. Ly D, Taylor JM, Tsatsaronis JA, et al. Plasmin(ogen) acquisition by group A Streptococcus protects against C3b-mediated neutrophil killing. J Innate Immun 2014;6:240-50.

5. Chung MC, Tonry JH, Narayanan A, et al. Bacillus anthracis interacts with plasmin(ogen) to evade C3b-dependent innate immunity. PLoS One 2011;6:e18119.

6. Cole JN, McArthur JD, McKay FC, et al. Trigger for group A streptococcal M1T1 invasive disease. FASEB J 2006;20:1745-7.

7. Mu Q, Kirby J, Reilly CM, et al. Leaky Gut As a Danger Signal for Autoimmune Diseases. Front Immunol 2017;8:598.

8. Antalis TM, Shea-Donohue T, Vogel SN, et al. Mechanisms of disease: protease functions in intestinal mucosal pathobiology. Nat Clin Pract Gastroenterol Hepatol 2007;4:393-402.

9. Zhang L, Seiffert D, Fowler BJ, et al. Plasminogen has a broad extrahepatic distribution. Thromb Haemost 2002;87:493-501.

10. Smits WK, Lyras D, Lacy DB, et al. Clostridium difficile infection. Nat Rev Dis Primers 2016;2:16020.

11. Seekatz AM, Young VB. Clostridium difficile and the microbiota. J Clin Invest 2014;124:4182-9.

12. Carter GP, Chakravorty A, Pham Nguyen TA, et al. Defining the Roles of TcdA and TcdB in Localized Gastrointestinal Disease, Systemic Organ Damage, and the Host Response during Clostridium difficile Infections. MBio 2015;6:e0551. 
13. Wu F, Wu J, Nicholson AD, et al. Tissue-type plasminogen activator regulates the neuronal uptake of glucose in the ischemic brain. J Neurosci 2012;32:9848-58.

14. Carter GP, Lyras D, Allen DL, et al. Binary toxin production in Clostridium difficile is regulated by CdtR, a LytTR family response regulator. Journal of Bacteriology 2007;189:7290-7301.

15. Cain JA, Dale AL, Niewold P, et al. Proteomics Reveals Multiple Phenotypes Associated with N-linked Glycosylation in Campylobacter jejuni. Mol Cell Proteomics 2019;18:715-734.

16. Law RH, Caradoc-Davies T, Cowieson N, et al. The X-ray crystal structure of fulllength human plasminogen. Cell Rep 2012;1:185-90.

17. Sorg JA, Dineen SS. Laboratory maintenance of Clostridium difficile. Curr Protoc Microbiol 2009; Chapter 9:Unit9A 1.

18. Lyras D, O'Connor JR, Howarth PK, et al. Toxin B is essential for virulence of Clostridium difficile. Nature 2009;458:1176-9.

19. Stabler RA, Dawson LF, Phua LT, et al. Comparative analysis of BI/NAP1/027 hypervirulent strains reveals novel toxin B-encoding gene $(t c d B)$ sequences. J Med Microbiol 2008;57:771-5.

20. Carter GP, Douce GR, Govind R, et al. The Anti-Sigma Factor TcdC Modulates Hypervirulence in an Epidemic BI/NAP1/027 Clinical Isolate of Clostridium difficile. PLoS Pathog 2011;7:e1002317.

21. Squire MM, Carter GP, Mackin KE, et al. Novel molecular type of Clostridium difficile in neonatal pigs, Western Australia. Emerg Infect Dis 2013;19:790-2.

22. Richards M, Knox J, Elliott B, et al. Severe infection with Clostridium difficile PCR ribotype 027 acquired in Melbourne, Australia. The Medical Journal of Australia 2011;194:369-371.

23. Dove $\mathrm{CH}$, Wang SZ, Price SB, et al. Molecular characterization of the Clostridium difficile toxin A gene. Infect Immun 1990;58:480-8.

24. Smith CJ, Markowitz SM, Macrina FL. Transferable tetracycline resistance in Clostridium difficile. Antimicrobial Agents and Chemotherapy 1981;19:997-1003.

25. Hutton ML, Cunningham BA, Mackin KE, et al. Bovine antibodies targeting primary and recurrent Clostridium difficile disease are a potent antibiotic alternative. Sci Rep 2017;7:3665.

26. Speir M, Lawlor KE, Glaser SP, et al. Eliminating Legionella by inhibiting BCL-XL to induce macrophage apoptosis. Nat Microbiol 2016;1:15034.

27. Nguyen TN, Padman BS, Usher J, et al. Atg8 family LC3/GABARAP proteins are crucial for autophagosome-lysosome fusion but not autophagosome formation during PINK1/Parkin mitophagy and starvation. J Cell Biol 2016;215:857-874.

28. Sorg JA, Sonenshein AL. Inhibiting the initiation of Clostridium difficile spore germination using analogs of chenodeoxycholic acid, a bile acid. J Bacteriol 2010;192:4983-90.

29. Wu G, Mazzitelli BA, Quek AJ, et al. Tranexamic acid is an active site inhibitor of urokinase plasminogen activator. Blood Adv 2019;3:729-733.

30. Quek AJH, Mazzitelli BA, Wu G, et al. Structure and Function Characterization of the a1a2 Motifs of Streptococcus pyogenes M Protein in Human Plasminogen Binding. J Mol Biol 2019;431:3804-3813.

31. Mileto SJ, Jarde T, Childress KO, et al. Clostridioides difficile infection damages colonic stem cells via TcdB, impairing epithelial repair and recovery from disease. Proc Natl Acad Sci U S A 2020;117:8064-8073.

32. Gonzalez-Miguel J, Siles-Lucas M, Kartashev V, et al. Plasmin in Parasitic Chronic Infections: Friend or Foe? Trends Parasitol 2016;32:325-335. 
33. !!! INVALID CITATION !!! 5.

34. Hervio LS, Coombs GS, Bergstrom RC, et al. Negative selectivity and the evolution of protease cascades: the specificity of plasmin for peptide and protein substrates. Chem Biol 2000;7:443-53.

35. Steinmetzer T, Pilgram O, Wenzel BM, et al. Fibrinolysis Inhibitors: Potential Drugs for the Treatment and Prevention of Bleeding. J Med Chem 2019.

36. Draxler DF, Sashindranath M, Medcalf RL. Plasmin: A Modulator of Immune Function. Semin Thromb Hemost 2017;43:143-153.

37. Herren T, Burke TA, Jardi M, et al. Regulation of plasminogen binding to neutrophils. Blood 2001;97:1070-8.

38. Yu H, Chen K, Sun Y, et al. Cytokines Are Markers of the Clostridium difficileInduced Inflammatory Response and Predict Disease Severity. Clin Vaccine Immunol 2017;24.

39. Goossens PL, Tournier JN. Crossing of the epithelial barriers by Bacillus anthracis: the Known and the Unknown. Front Microbiol 2015;6:1122.

40. Meinel C, Sparta G, Dahse HM, et al. Streptococcus pneumoniae From Patients With Hemolytic Uremic Syndrome Binds Human Plasminogen via the Surface Protein PspC and Uses Plasmin to Damage Human Endothelial Cells. J Infect Dis 2018;217:358-370.

41. Barthel D, Schindler S, Zipfel PF. Plasminogen is a complement inhibitor. J Biol Chem 2012;287:18831-42.

42. Stansfield R, Morris D, Jesulola E. The Use of Tranexamic acid (TXA) for The Management of Haemorrhage In Trauma Patients In The Prehospital Environment: Literature Review and Descriptive Analysis of Principal Themes. Shock 2019.

Author names in bold designate shared co-first authorship. 
A

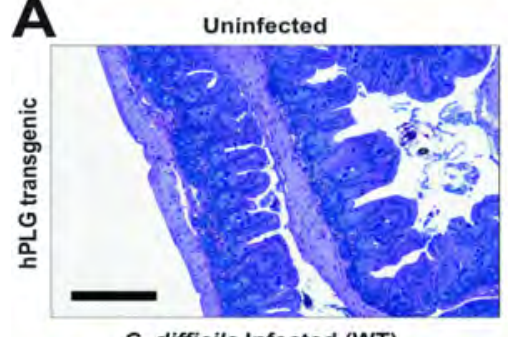

C difficile Infected (WT)

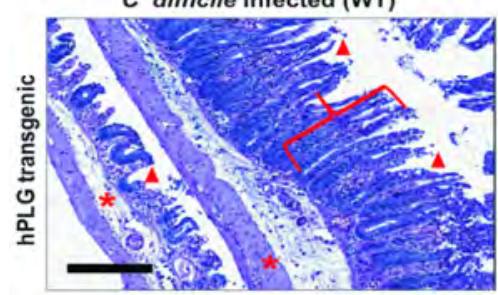

Uninfected

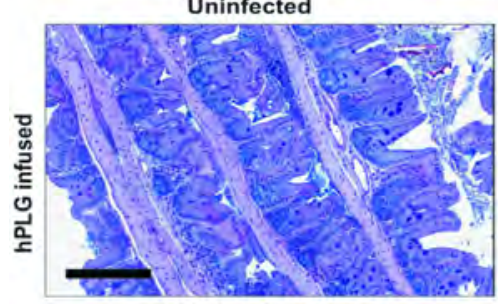

C difficile Infected (WT)

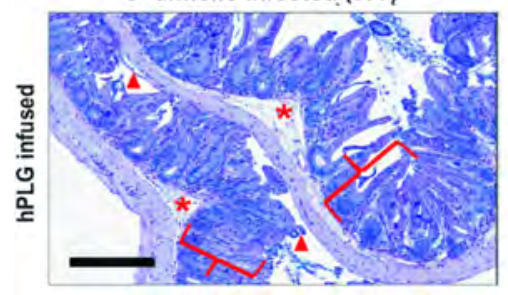

C difficile Infected (A-B-)

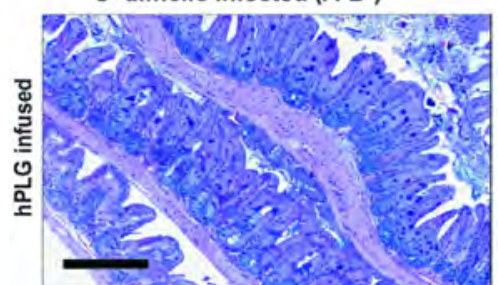

B

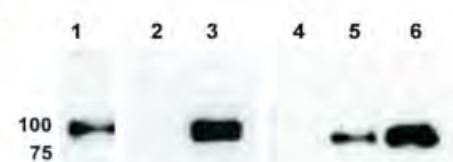

D

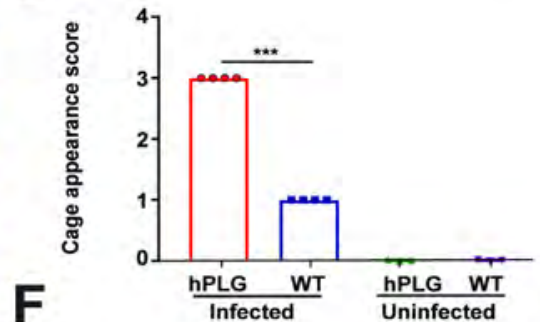

F

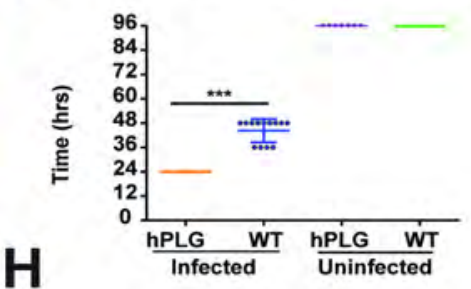

H

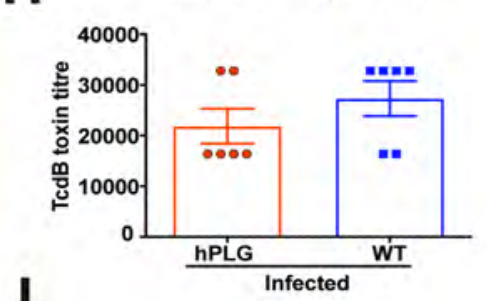

J

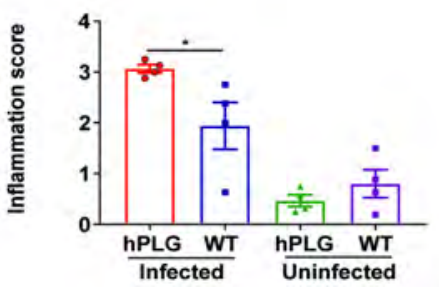

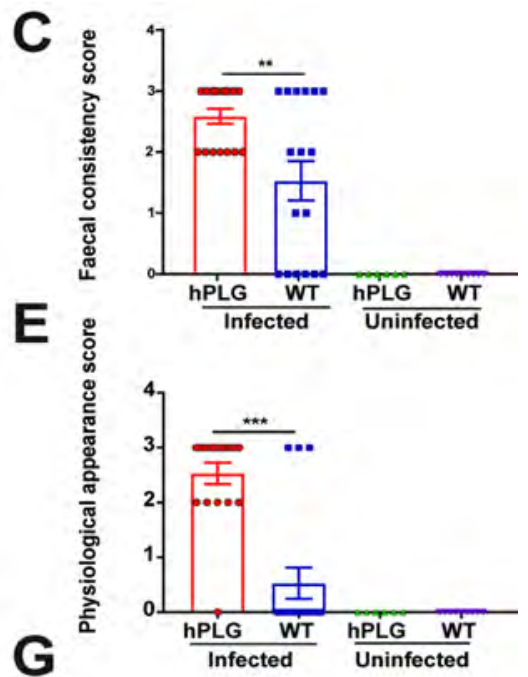
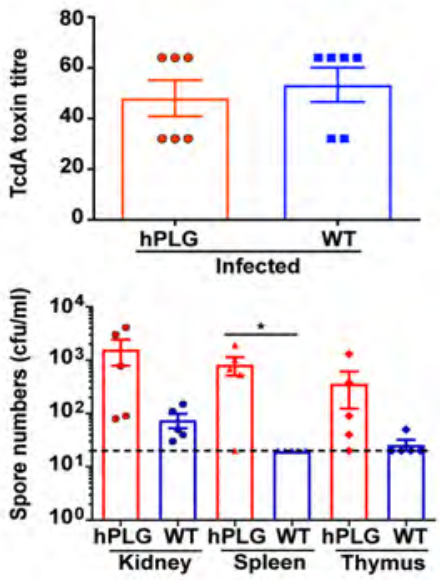

K

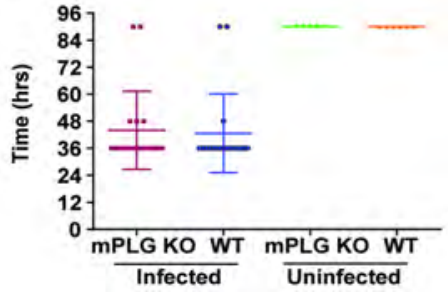




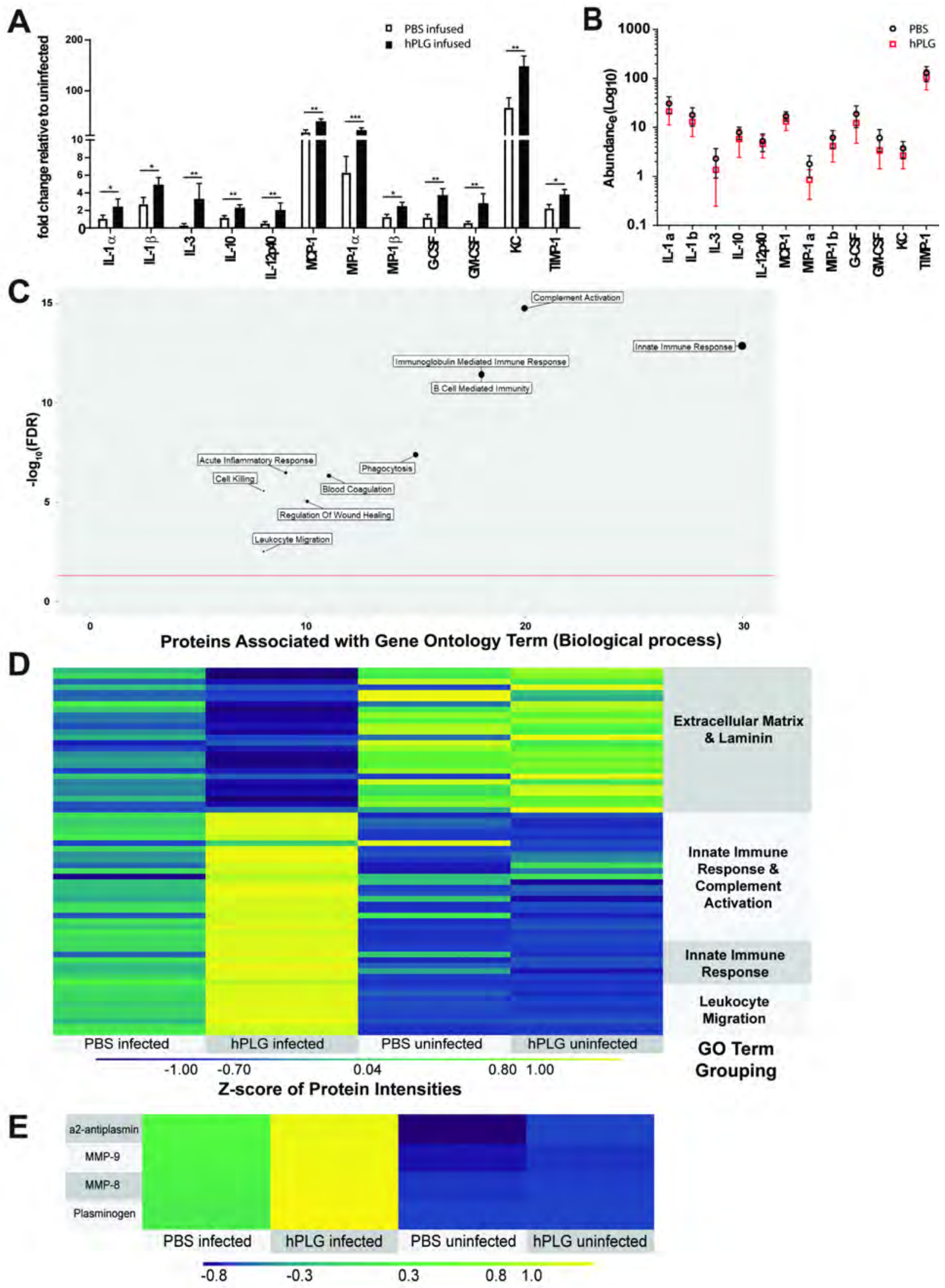




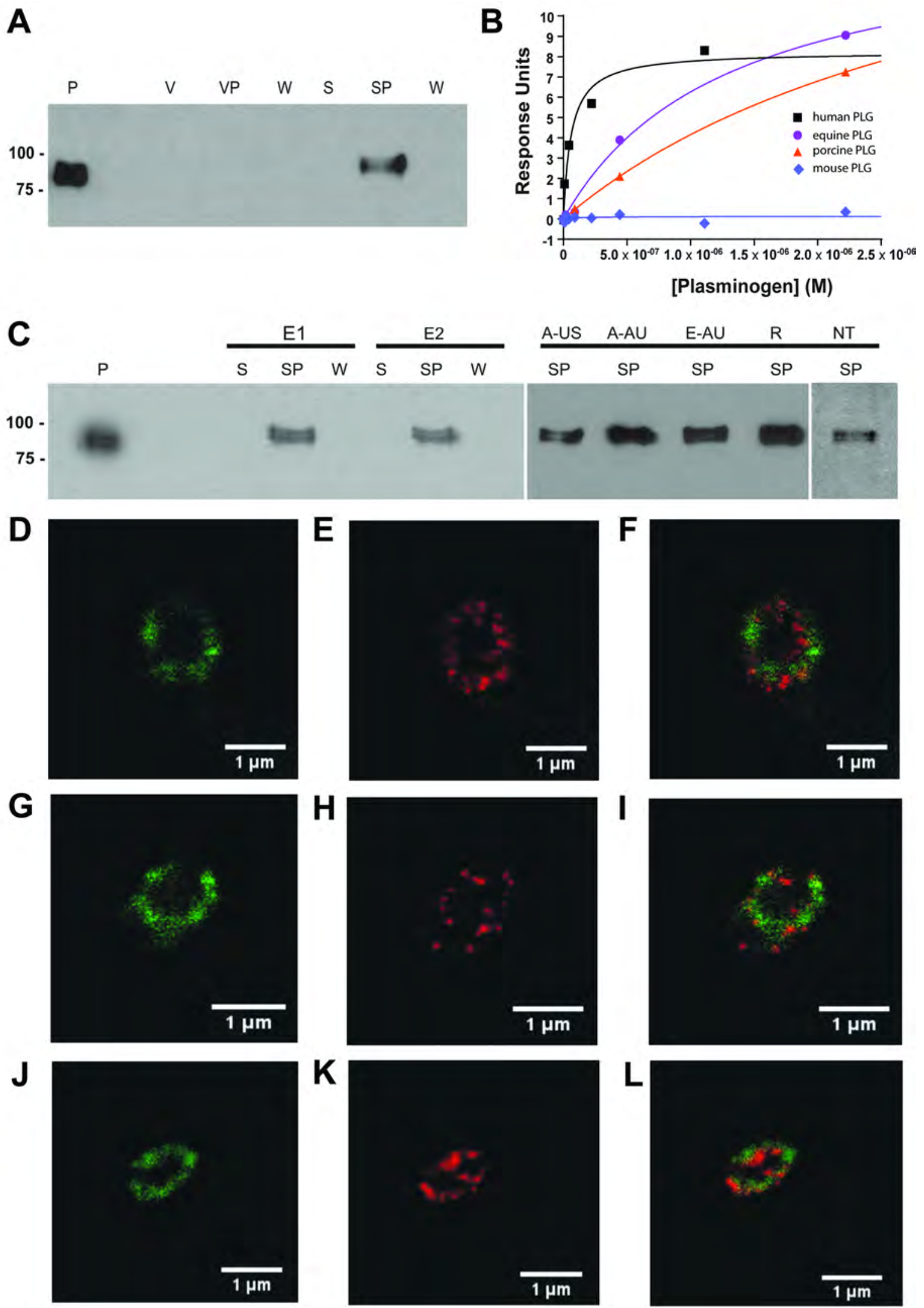
A

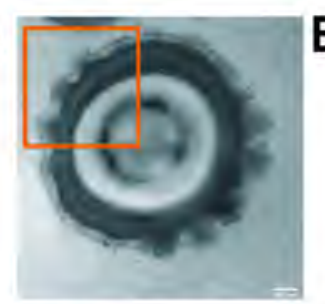

E

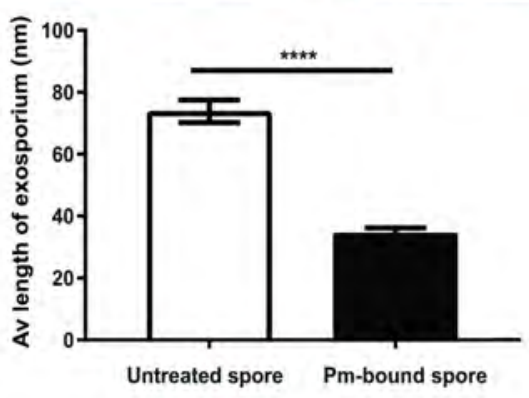

G

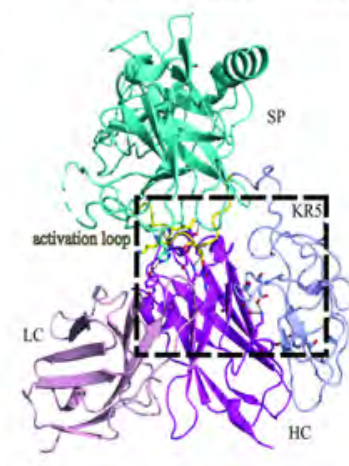

I

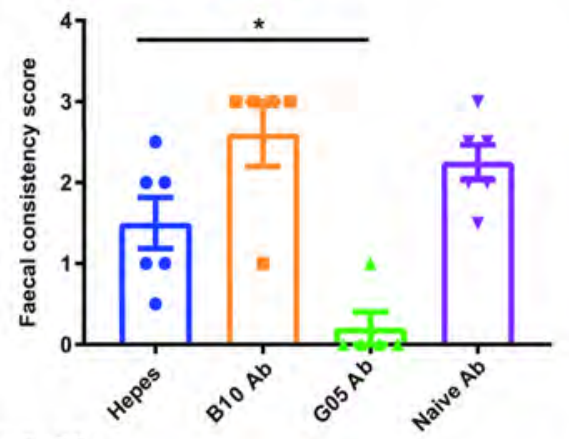

K

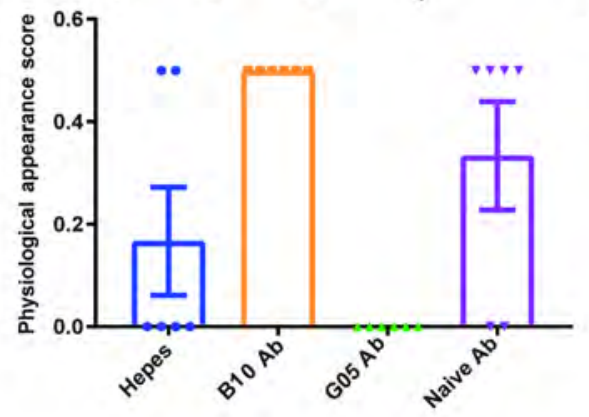

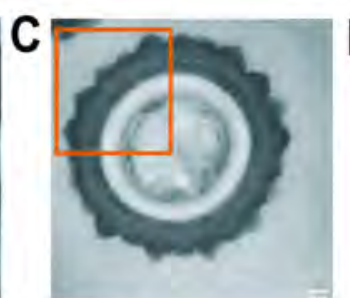

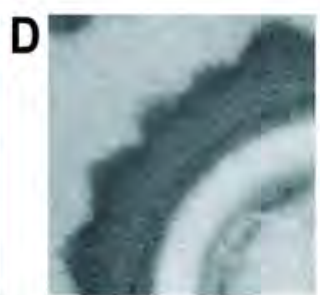

F

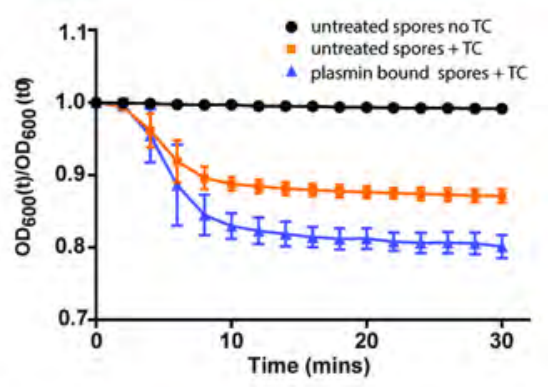

H
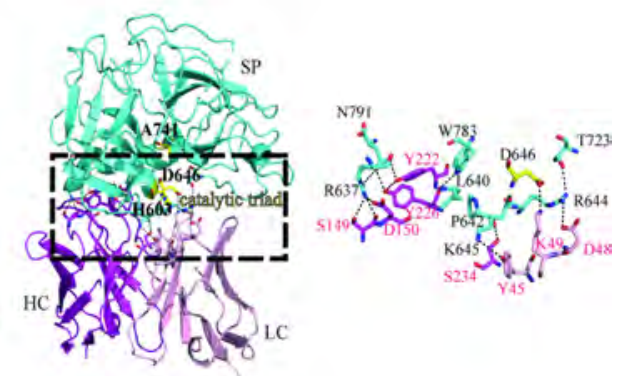

J

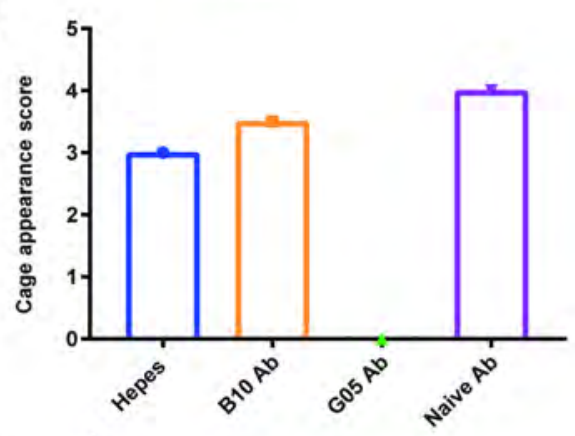

L

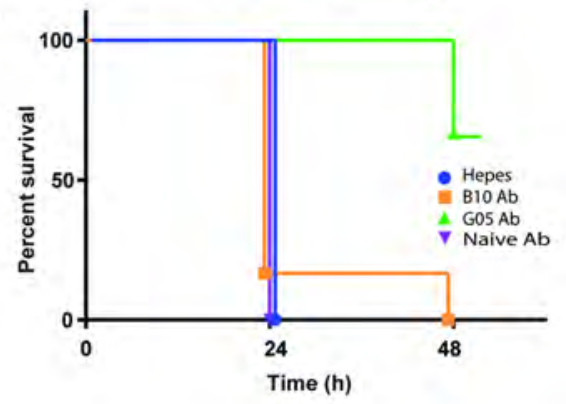




\section{What you need to know:}

Plasmin(ogen) is a protease that is required for wound healing. This study investigated its effects on gastrointestinal damage resulting from infection with enteric pathogens such as Clostridioides difficile.

NEW FINDINGS: Human plasmin(ogen), recruited to the damaged gut of $C$ difficile-infected mice, increases disease severity; this effect is neutralized by an antibody against activated plasminogen. Plasmin(ogen) also altered the spore surface, accelerating germination.

LIMITATION: These studies were conducted in mice. Studies of plasmin activation inhibition using antibody therapy are needed in humans.

IMPACT: Proteases might accelerate progression of disease following infection with $C$ difficile or other enteric pathogens. Inhibitors of plasminogen activation might be used to reduce the severity of $C$ difficile-mediated disease.

\section{Lay summary}

The human protease plasmin(ogen) is recruited to the toxin-damaged gut during infection with pathogenic bacteria. Plasminogen can alter the bacterial spore surface and promote germination, and exacerbates inflammation and disease, promoting spore dissemination. 